\title{
The Cremaster in Undescended Testis
}

\author{
Salvatore Arena Donatella Di Fabrizio Pietro Impellizzeri Carmelo Romeo \\ Department of Human Pathology of Adult and Childhood "Gaetano Barresi", Unit of Pediatric Surgery, University of \\ Messina, Messina, Italy
}

Dear Editor,

We read and truly appreciated the recent interesting article written by Molinaro et al. [1], focusing on histomorphometric changes and immunoexpression of myosin isoforms in cremasteric muscle (CM) of pediatric patients affected by undescended and retractile testis. Even if still unclear, the role of CM in the pathogenesis of undescended testis has been appreciably investigated in the last few years. CM is characteristically composed of both smooth muscle and striated cells, the latter featuring multifocal innervation by multiple neuromuscular innervations [1]. In their study, authors documented a thinning of CM fibers, whose statistical significance increases with age of patients affected by undescended testis, and an immunohistological pattern of "hybrid fiber" co-expressing multiple $\mathrm{MyHC}$ isoforms, both suggesting muscular denervation [1].

Previous reports showed a nonsignificant difference in the diameter of type 1 fibers (fibers staining strongly for myofibrillary ATPase reactions after acid preincubation, expressing slow myosin heavy chain) and fiber 2 (fibers staining strongly for myofibrillary ATPase reactions after alkaline preincubation, expressing fast myosin heavy chain) in the cremaster of congenital orthotopic testis, while type 2 fibers were observed as significantly thinned in the CM of patients affected by undescended testis [2]. These data, together with electromyographic evaluation of cremaster reflex showing a segmental or suprasegmental lesion, reflect an alteration in the corticospinal tracts of boys affected by undescended testis [2]. These data are strongly in accordance with clinical evidence of the prevalence of undescended testis in children affected by cerebral palsy at least 10-fold greater than that of the general population [3].

Molinaro et al.'s [1] study elegantly confirms the hypothesis of an impaired innervation of cremaster as a causative role in the pathogenesis of undescended testis. We believe it is worth better defining whether the altered innervation in cremaster of undescended affected patients is at corticospinal or at nucleus level of the genitofemoral nerve and, then, the role of innervation on the abnormal arrangement of the cytoskeleton of CM cells in these patients [4]. Finally, we would like to congratulate Molinaro et al. [1] for their remarkable article, highlighting the obscure pathogenesis of undescended testis.

\section{Conflict of Interest Statement}

The authors have no conflicts of interest to declare. karger@karger.com

www.karger.com/uin

Karger!"
(C) 2021 S. Karger AG, Basel

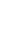

Salvatore Arena

Department of Human Pathology of Adult and Childhood "Gaetano Barresi" Unit of Pediatric Surgery, University of Messina, Viale Gazzi-AUO “Gaetano Martino” IT-981 25 Messina (Italy)

salarena@unime.it 


\section{Funding Sources}

The authors do not have funding to disclose.

\section{Author Contributions}

S.A. and D.D.F. conceived of the presented idea and wrote the letter with input of all authors; P.I. and C.R. supervised the findings of the work.

\section{References}

1 Molinaro F, Fusi G, Aglianò M, Volpi N, Franci D, Lorenzoni P, et al. Clinically differentiated abnormalities of the architecture and expression of myosin isoforms of the human cremaster muscle in cryptorchidism and retractile testis. Urol Int. 2020;16:1-11.
2 Tanyel FC, Erdem S, Altunay H, Ergün L, Ozcan Z, Alabay B, et al. Distribution and morphometry of fiber types in cremaster muscles of boys with inguinal hernia or undescended testis. Pathol Res Pract. 2000;196(9):613-7.

3 Barthold JS, Wintner A, Hagerty JA, Rogers KJ, Hossain MJ. Cryptorchidism in boys with cerebral palsy is associated with the severity of disease and with co-occurrence of other congenital anomalies. Front Endocrinol. 2018;9: 151.
4 Arena S, Favaloro A, La Face A, Romeo P, Scalfari G, Turiaco N, et al. Altered integrins expression of patients affected by cryptorchidism. Urol Int. 2018;101(2):219-23. 